\title{
NUMERICAL SIMULATION OF FILM COOLING A TURBINE BLADE THROUGH A ROW OF HOLES
}

\author{
Djamel Cherrared ${ }^{1}$ \\ Keywords: Film Cooling, Turbulent Flows, Numerical Simulation, Cooling Effectiveness
}

\begin{abstract}
We undertake a numerical three-dimensional study of the interaction of a row of discrete jets in a wall with a transversal compressible flow for different injection rate $(\mathrm{M}=0.3,0.5,0.7$ and 1.4). This simulation is applied to the stator blade of the CFM56 engine and is performed using the computational fluid dynamics (CFD) simulation tool, with CFX.13 software. Reynolds averaged Navier-Stokes equations were solved using a finite volume method. Turbulence closure was achieved using the Shear-Stress Transport model (SST). The velocity and temperature distributions and the film cooling effectiveness are presented and discussed. We found that the best cooling effectiveness occurs at $M=0.7$. More, for higher injection rate $(M=1.4)$, the results show the existence of two counter- rotating vortices. These vortices transport the hot gas in the jet and thus degrade the protective wall.
\end{abstract}

\section{INTRODUCTION}

It is well known from thermodynamic analysis that the performance of a gas turbine is strongly influenced by the temperature at inlet to the turbine. Modern gas turbine engines are designed to operate at inlet temperatures of $1800-2200 \mathrm{~K}$ which are far beyond the allowable metal temperatures. Thus, to maintain acceptable life and safety standards, the structural elements need to be protected against the severe thermal environment. This calls for an efficient cooling system. One such cooling technique currently used for high temperature turbines, is film cooling.

The interaction of cool air jets with hot cross-flow fields is an interesting topic of research and occurs in a variety of industrial applications: pollutant discharges, vertical takeoff and landing (VTOL) engineering and aerodynamics, jets into combustors, waste disposal from smoke stacks into the atmosphere, film cooling of turbine blades, etc.

Discrete jet film-cooling is one of the techniques used to protect the blades that are particularly thermally exposed (see Fig. 1). The best compromise between admissible metal temperature and aerodynamic efficiency becomes a major objective in cooled blade design. In this technique, cooler air is injected into the high temperature boundary layer on the blade surface. Since the injected cooler air is bled directly from the compressor before it passes through the combustion chamber, it represents a loss in the total power output.

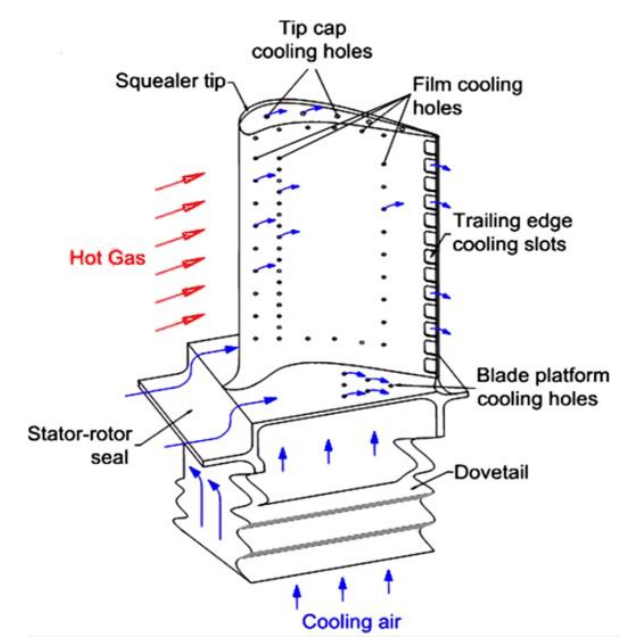

Figure 1. Gas turbine blade cooling schematic 
A considerable effort has been devoted to understanding the coolant film behavior and its interaction with the mainstream flow (Chernobrovkin and Lakshminarayana [1], Hoda and Acharya [2]).The film cooling performance is influenced by the wall curvature, three dimensional external flow structures, free-stream turbulence, compressibility flow unsteadiness, the injection rate, the angle of injection and the geometry of the hole (Gartshore et al. [3]). Among these parameters, the inclination angle $\alpha$ and the injection rate ratio $\mathrm{M}$ are extremely important, since they control the vertical penetration and the lateral spreading of the jet, which in turn determine the cooling efficiency. Analysis of the discrete-film-cooling performance requires an understanding of the fundamental jet-in-crossflow. The jet-in-crossflow problem has been investigated for over fifty years, and has been discussed by Goldstein [4] and Margason [5]. Previous research on film-cooling performance has tended to focused on flat plate models with various injection row arrangements and different shapes of cooling holes. Foster and Lampard [6] presented laterally averaged film effectiveness results over a flat plate with inclination angles $35^{\circ}, 55^{\circ}$ and $90^{\circ}$ for long injection tubes spaced at three-diameter apart. The authors have measured only the film cooling effectiveness at blowing ratio (injection rate) of $\mathrm{M}=0.5$ and 1.4 , and did not measure the heat transfer coefficient distributions. Nasir et al. [7] studied the effect of compound angle injection on flat surface film cooling with large stream-wise angle. Film cooling measurements are presented over a flat surface through a single row of discrete holes angled $55^{\circ}$ along the stream-wise direction. The holes are angled to $0^{\circ}$ and $60^{\circ}$ in the lateral direction to study the effect of compound angle injection. Film cooling effectiveness is generally lower for a large stream wise angle of $55^{\circ}$ compared to the typically used shallow angle of $35^{\circ}$. The compound angle of $60^{\circ}$ provides significantly higher film cooling effectiveness than the simple injection case but also causes much higher heat transfer coefficients. Overall, the compound angle injection with large stream wise angle holes provide improved performance compared to simple angle injection geometry. CFD simulation is also an important method to study film cooling effectiveness and flow field of jet in crossflow in recent years. Xiao et al. [8] investigated the mixing characteristics of jet in crossflow using the CFD code Fluent. The three-dimensional mean and fluctuating characteristics of an impinging density jet in a confined crossflow were numerically investigated using the RNG turbulence model by Fan et al. [9]. Hoda and Acharya [2] investigated the performance of several existing turbulence models for the prediction of film cooling jet in a crossflow. Fadéla et al. [10] have used the Reynolds Stress Model for the prediction of film cooling at the leading edge of a Symmetrical turbine blade. The performance of selected model has been compared to that of the standard k- $\varepsilon$ model.

Bons et al. [11] studied the effect of high stream turbulence on film cooling effectiveness. At high free stream turbulence, heat transfer coefficients with film cooling are not as significantly as the film effectiveness. Film injection by itself produces high heat transfer coefficient enhancement due to high turbulent mixing between jet mainstream.

Film cooling is injected near the blade surface through the holes thereby forming a layer of cool fluid between the hot gases (air) and the blade surface. The working principle of film cooling is sketched in Fig 2 for injection of the coolant from a row of holes. The ejected cool air forms an insulating layer between the hot main flow and the surface to be cooled, reducing thereby the heat transfer to the surface.

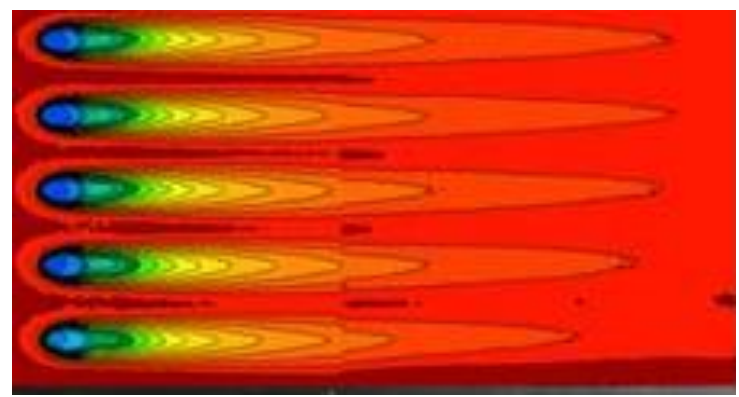

Figure 2. Visualization of film cooling

The present paper contributes to the development of a prediction method for the simulation and understanding the coolant film behavior and its interaction with the crossflow. The study of film cooling is applied on a fixed blade of a sector HPT of the CFM56-7B engine. The application of computational fluids dynamics to film cooling problem is assessed in this study. Results are presented in terms of temperature and 
velocity calculation in the flow field. The objective is to evaluate the impact of jet hole arrangement on the filmcooling effectiveness. This is determined by using the CFD technique.

The mainstream turbulence level fixed at 1\%. The density ratio is assumed 1 but the tested blowing ratio is increased from 0.3 to 1.4 .

\section{PROBLEM DESCRITION AND COMPUTATIONAL MODEL}

Figure 3 shows a section of the blade to better visualize the injections holes configuration. We consider a row of holes inclined at an angle compound and located on the extrados of the blade. A blade for an axial-flow turbine includes an intrados producing a positive pressure between a leading edge and a trailing edge, and an extrados producing a negative pressure. The intrados is formed at its rear portion with a flat surface portion connected to the trailing edge, and the extrados has a curved surface portion formed at least at a portion corresponding to the flat surface portion. The trailing edge of the turbine blade is pointed at its end.

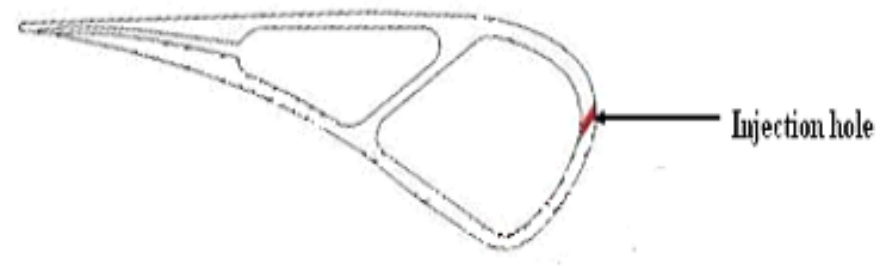

Figure 3. Geometry of the blade

The blade is $67 \mathrm{~mm}$ length and $20.8 \mathrm{~mm}$ in maximum width, it is provided with injection holes of diameter $\mathrm{D}=0.6 \mathrm{~mm}$. The geometry of the configuration injection holes studied here is shown in Table 1 .

Table 1. Geometric parameters

\begin{tabular}{|c|c|c|c|c|}
\hline Configuration & $\alpha$ & $\beta$ & D[mm] & Z/D \\
\hline & 60 & 3 & 0.6 & 3.8 \\
\hline
\end{tabular}

The compound angle injection hole has two injection angles as shown in Figure 4. Inclination angle $(\alpha)$ is defined as the angle between injection vector and its projection on the $\mathrm{x}-\mathrm{z}$ plane, whereas orientation angle $(\beta)$ is defined as the angle between streamwise direction and the projection of injection vector on the $\mathrm{x}-\mathrm{z}$ plane. In a compound angle orientation system, coolant is injected with a spanwise momentum, which provides more uniform film coverage and shows higher heat transfer coefficient enhancement.

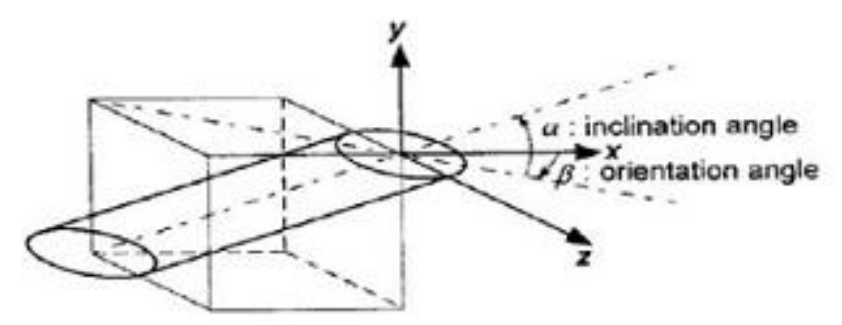

Figure 4. Compound hole Configuration

The approach velocity of the main flow is about $100 \mathrm{~m} / \mathrm{s}$ with a temperature of $1750 \mathrm{~K}$.Tthis values are the nominal design conditions [12]. We start the work by considering a single injection rate of $M=0.5$. It is calculated by the following equation:

$$
M=\frac{\bar{\rho}_{c} \tilde{U}_{c}}{\bar{\rho}_{\infty} \tilde{U}_{\infty}}
$$

Where the subscript (c) indicates the density and velocity of the jet and the index ( $\infty)$ refers to the conditions of the main flow. 
The jet velocity injected by the tube is calculated using the injection rate $\mathrm{M}$ with a temperature of 804 $\mathrm{K}$. These temperatures (coolant and crossflow) correspond to the operating conditions of a gas turbine (Halila et al. [12]). Several options exist to specify the turbulence quantities at Inlet (crossflow and jet). We selected the medium option, which fixes the turbulence intensity at $1 \%$ for crossflow and the high option, which fixes the turbulence intensity at $10 \%$ for jet. Subsequently, we vary the injection rate as $(\mathrm{M}=0.3,0.5,0.7$ and 1.4$)$.

The main aerodynamic condition used in our simulations is the injection rate M. Four injection rates are tested. The boundary conditions imposed as well for the main flow for the cooling jet are summarized in the table below.

Table 2. Boundary conditions parameters

\begin{tabular}{|c|c|c|c|c|c|c|c|c|}
\hline & & \multicolumn{3}{|c|}{ Main flow (crossflow) } & \multicolumn{3}{|c|}{ Cooling flow (jet) } & $\rho_{d} / \rho_{c}$ \\
\hline \multirow[t]{5}{*}{ Case } & $M$ & $\mathrm{U}_{x}(\mathrm{~m} / \mathrm{s})$ & $\mathrm{Tu}_{\mathrm{u}_{0}}$ & $T_{\infty}(\mathrm{K})$ & $\mathrm{U}_{\mathrm{c}}(\mathrm{m} / \mathrm{s})$ & $\mathrm{Tu}_{\mathrm{e}}$ & $T_{c}(\mathrm{~K})$ & \multirow{5}{*}{1} \\
\hline & 0.3 & \multirow{4}{*}{100} & \multirow{4}{*}{$1 \%$} & \multirow{4}{*}{1750} & 30 & \multirow{4}{*}{$10 \%$} & \multirow{4}{*}{$80 t$} & \\
\hline & 0.5 & & & & 50 & & & \\
\hline & 0.7 & & & & 70 & & & \\
\hline & 1.4 & & & & $1+0$ & & & \\
\hline
\end{tabular}

So the injection rate $(\mathrm{M})$ is one dominant parameter in film cooling study. It represents the flux ratio between the crossflow and the coolant. In the present study, the air is used for the coolant and the crossflow. Therefore, the injection rate is essentially the velocity ratio between the mainstream and the injection air. The density ratio is nominally assumed to one $(\mathrm{DR} \approx 1)$.

\section{NUMERICAL PROCEDURE}

To start the analysis, we specially pay attention in selected grid. The numerical domain was discretized using an unstructured tetrahedral grid illustrated in figures 5 and 6.

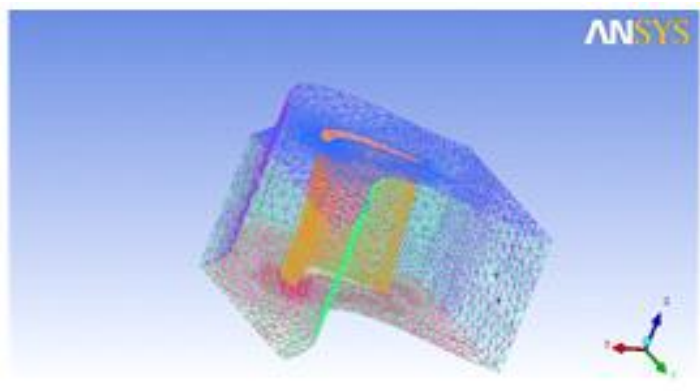

Figure 5. Computational geometry

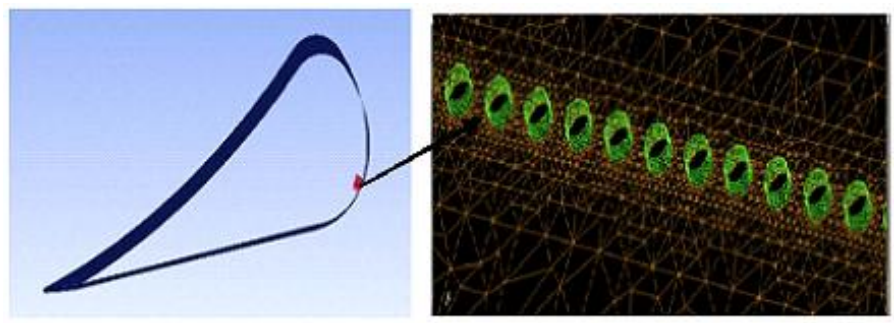

Figure 6. Mesh of the holes

Non uniform grid was generated and grid refinement close to the wall and injection hole zone was applied. Several successive grid refinements have been carried out in every case to get negligible effect of the mesh in the solutions. The mesh obtained is of 1891639 nodes and 9631278 elements. First, we generate the tetrahedral element on all geometry, thereafter we proceed to the refinement of blade and holes generating prism layers to better simulate the flow in the boundary layer. The number of tetrahedral elements is 9271755 , the number of prismatic element is 7625551 and the number of pyramidal elements is 799 .

This study uses a commercial CFD code based on the finite volume method, CFX. 13. The flow is considered steady state. The crossflow and the jet are considered the ideal gas. 
The Navier-Stokes equations may be decomposed into a mean part and a fluctuating part.

The Favre-averaged equations governing the fluid for mass, momentum and energy conservation transport are given below:

$$
\begin{gathered}
\frac{\partial}{\partial x_{i}}\left(\bar{\rho} \tilde{U}_{i}\right)=0 \\
\frac{\partial}{\partial x_{i}}\left(\bar{\rho} \tilde{U}_{i} \tilde{U}_{j}\right)=-\frac{\partial \bar{P}}{\partial x_{i}}+\frac{\partial}{\partial x_{i}}\left(\bar{\tau}_{i j}-\overline{\rho u_{i} u_{j}}\right) \\
\frac{\partial}{\partial x_{i}}\left(\bar{\rho} \tilde{U}_{i} \tilde{T}_{j}\right)=\frac{\tilde{U}_{i}}{C_{p}} \frac{\partial \bar{P}}{\partial x_{i}}+\frac{\partial}{\partial x_{i}}\left(\frac{\mu}{\operatorname{Pr}} \frac{\partial \tilde{T}}{\partial x_{i}}\right)+\frac{\overline{\tau_{i j}}}{C_{p}} \frac{\partial U_{j}}{\partial x_{i}}+\frac{\partial\left(-\overline{\rho u_{i} \theta}\right)}{\partial x_{i}}
\end{gathered}
$$

The turbulent Reynolds stress $-\overline{\rho u_{i} u_{j}}$ and turbulent heat fluxes $-\overline{\rho u_{i} \theta}$ require modelling in order to close the equations. The Boussinesq eddy-viscosity hypothesis is used to yield the turbulent stress. The turbulent heat fluxes will be modelled by means of simple eddy diffusivity and gradient diffusion type model.

$$
\begin{aligned}
-\overline{\rho u_{i} u_{j}}= & 2 \mu_{t}\left(\tilde{S}_{i j}-\frac{1}{3} \tilde{S}_{k k} \delta_{i j}\right)-\frac{2}{3} \bar{\rho} K \\
& -\overline{\rho u_{i} \theta}=\frac{\mu_{t}}{\operatorname{Pr}_{t}} \frac{\partial \tilde{T}}{\partial x_{i}}
\end{aligned}
$$

With $\mu_{t}$ is the turbulent eddy viscosity. $\operatorname{Pr}_{t}$ is the turbulent Prandtl number taken to be 0.9 .

The turbulent eddy viscosity is computed from:

$$
\mu_{t}=\frac{\rho a_{1} k}{\max \left(a_{1} \omega, \Omega F_{2}\right)}
$$

$\Omega$ is the vorticity magnitude. For more details on this model, refer to the reference [13].

The SST turbulence model with automatic near wall treatment is used to predict the flow structure and heat transfer over the film-cooling surface. This treatment exploits the simple and robust near wall formulation of the underlying $\mathrm{k}-\omega$ model and switches automatically from a low-Reynolds number formulation to a wall function treatment based on the grid density. The advantage is that the user can make optimal use of the advanced performance of the turbulence model, for a given grid. The automatic wall treatment avoids the deterioration of the results typically seen if low-Re models are applied on under-resolved grids.

The first layer of the elements on the wall and near the hole for all grids has $\mathrm{y}^{+}$below 1 .

The shear stress transport (SST) formulation combines the better of two worlds (k- $\varepsilon$ and $k-\omega)$. The use of a $k-\omega$ formulation in the inner parts of the boundary layer makes the model directly usable all the way down to the wall through the viscous sub-layer; hence the SST model can be used as a Low-Re turbulence model without any extra damping functions. The SST model often merits it for its good behavior in adverse pressure gradients and separating flow.

The governing equations are iteratively solved by the finite volume method. The convection term in the momentum equation are approximated by the second-order upwind scheme [14]. In order to avoid temperature over- or undershoots, the convection term of the energy equation is discretized by the third-order QUICK scheme [15]. The QUICK scheme involves a third-order accurate upwind differencing, which possesses the stability of the first-order upwind formula and is free for the substantial numerical diffusion experienced with the usual first-order schemes. The coupling between velocity and pressure in momentum equations is governed by the SIMPLE scheme. The SIMPLE algorithm uses a relationship between velocity and pressure corrections to enforce mass conservation and to obtain the pressure field. The CFX solver finishes the calculations when the equation residuals calculated using the specified method is below the target residual value. A convergence criterion of $10^{-4}$ is used to ensure negligibly small iteration errors. 


\section{RESULTS AND DISCUSSION}

Figure 7 represents the temperature contours (isotherms) at various X/D with an injection rate of 0.5 .

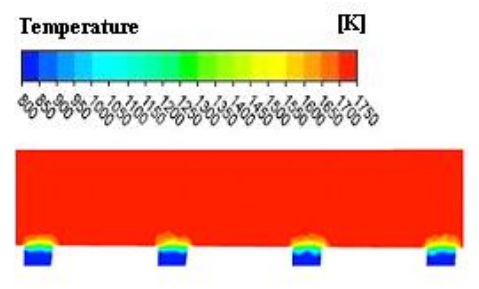

$X / D=0$

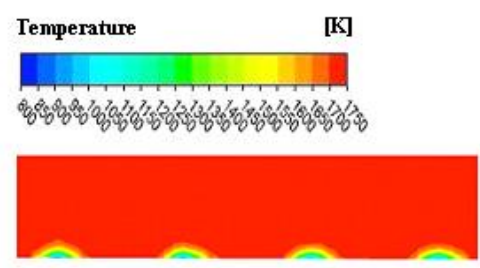

$X / D=1$

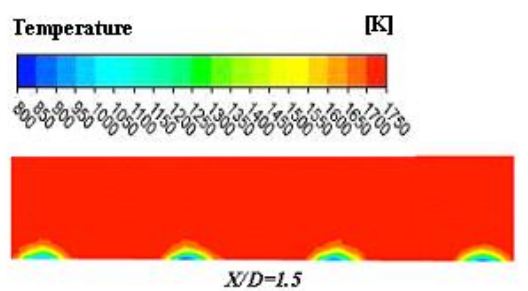

Figure 7. Representation of efficiency contours at vertical planes along Z/D

This figure illustrates the temperature distribution of the jets. It shows the interaction between hot gases (main flow) and the cooling air (jet). The jet is uniform and presents a symmetrical appearance for the same row holes. We notice a boundary layer thicker at the injection point for which the thickness decreases in moving away from this point, which is explained by the dilution of jet in the main flow.

The details of the jet interaction with the main flow near the injection are illustrated in Figure 8 by considering the velocity vectors at $\mathrm{X} / \mathrm{D}=0,1$ and 1.5 in the transverse plane $(\mathrm{Y} / \mathrm{D}, \mathrm{Z} / \mathrm{D})$ for an injection rate of $\mathrm{M}=0.5$.The results do not show the development of large vortex structure. These vortexes bring more hot gas in the jet and degrade the protective plate. The jets extend over a certain height and weaken unless $X / D=1.5$, and no interaction has been occurred between the jets.

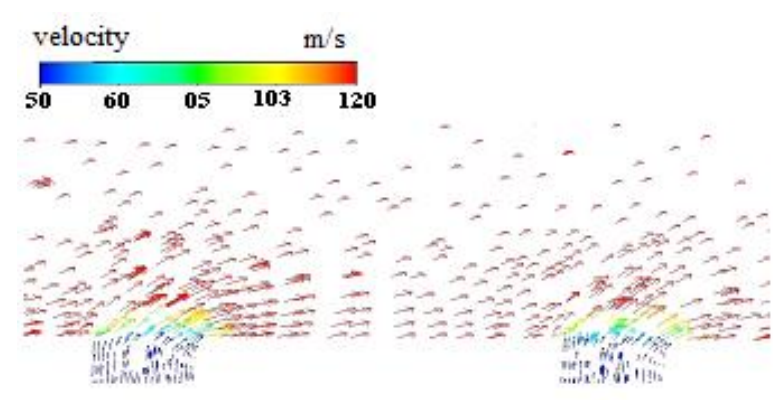

$X / D=0$
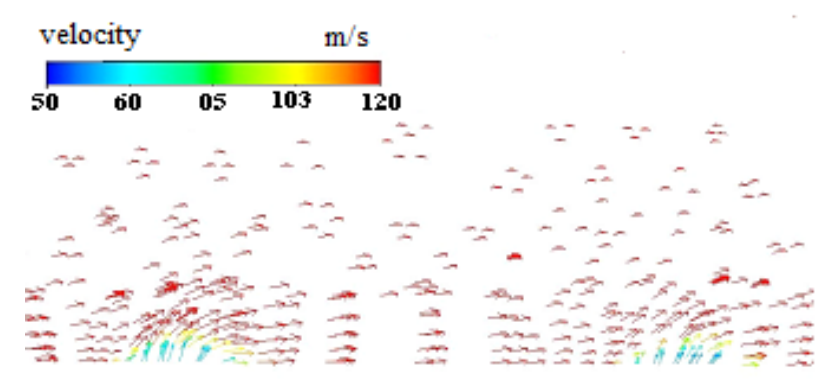

$X / D=1$

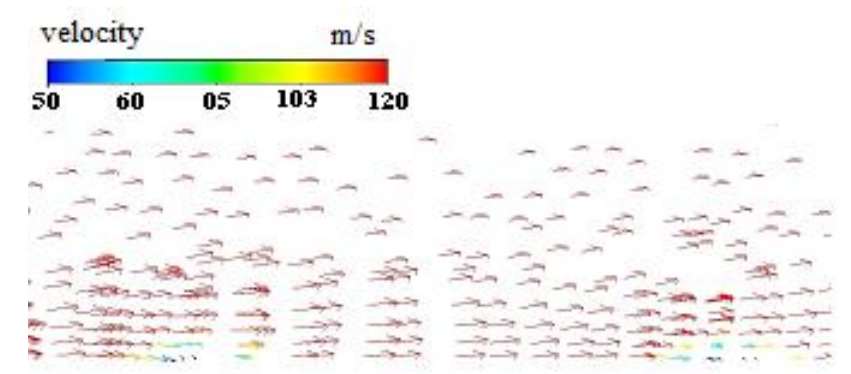

$X / D=1.5$

Figure 8. Computed velocity vectors at vertical planes along Z/D direction 
Figure 9 shows the velocity and temperature fields, on a plane passing through the injection hole for an injection rate of $M=0.5$. These figures show the interaction between the jet and the crossflow. They illustrate the detachment of the jet and its reattachment further downstream.
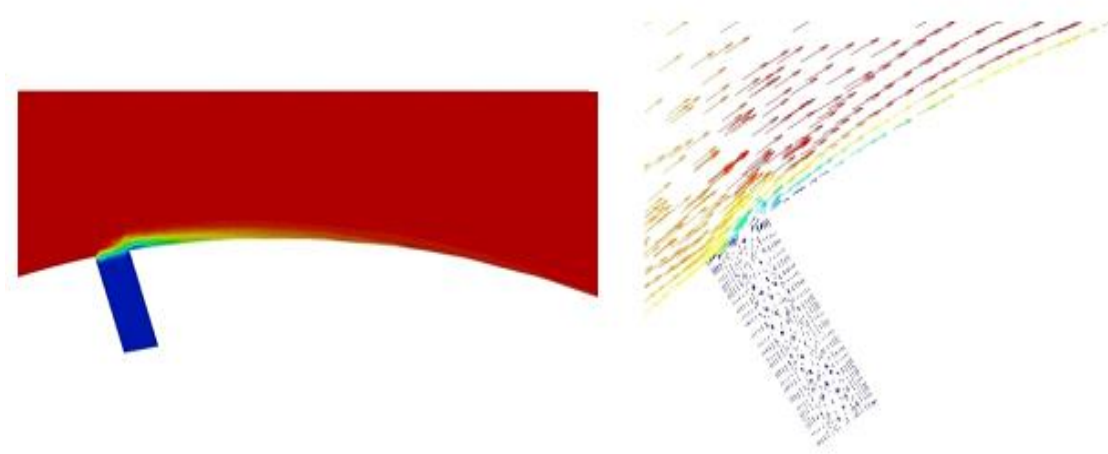

Figure 9. The fields of velocity and temperature

Figure 10 shows the distribution of temperature on the wall of the blade for an injection rate of $\mathrm{M}=0.5$. The jet extends over a large portion of the blade. We have a uniform cooling for the entire row of holes. However, cooling is still insufficient; a portion of the blade is improperly cooled. To improve the cooling, we varied the ratio of injection to investigate the cooling efficiency on this part.
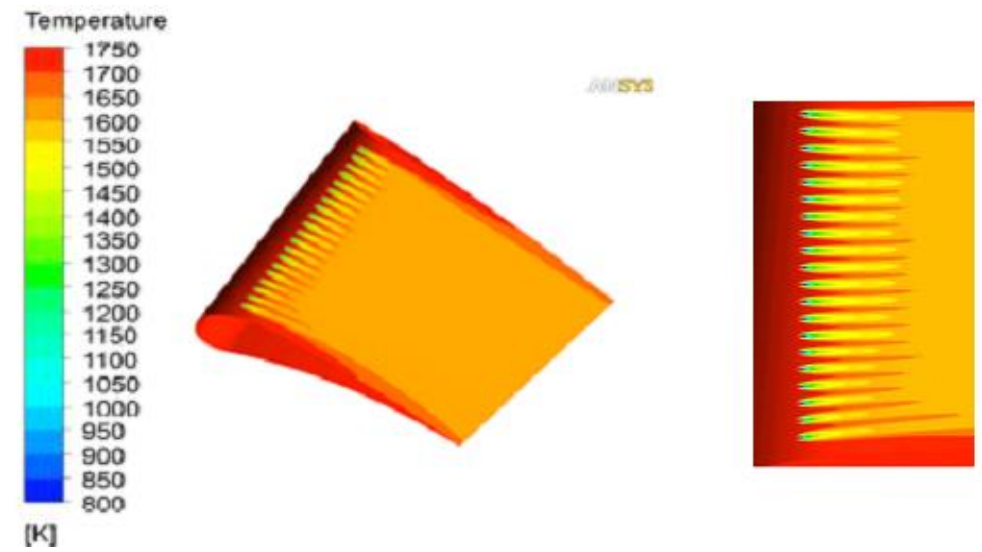

Figure 10. Temperature distribution

The presence of flow at different temperatures leads to introduce an effectiveness parameter compared to the adiabatic temperature. We define the average effectiveness of film cooling by:

$$
\eta=\frac{\tilde{T}_{\infty}-\tilde{T}_{a d}}{\tilde{T}_{\infty}-\tilde{T}_{c}}
$$

Where the subscript (c) indicates the temperature of the jet, (ad) the adiabatic temperature of the wall and the index $(\infty)$ refers to the conditions of the main flow. $\mathrm{T}_{\mathrm{ad}}$ is calculated at the center of the injection hole from $\mathrm{X} / \mathrm{D}=0$ to $\mathrm{X} / \mathrm{D}=7$

The cooling efficiency takes the value 1 if the temperature is equal to that of the jet (maximum cooling) and 0 if it is equal to that of the main flow (no cooling).

Figure 11 shows the longitudinal distribution of the average cooling effectiveness for the four injection rate $(\mathrm{M}=0.3,0.5,0.7$ and 1.4).

We first observe that the effectiveness for the four injection rate has well decreased. However, analysis of graphs permits to highlight two main zones. In the zone $\mathrm{X} / \mathrm{D}=[0,1.3]$ illustrated in Figure 11, we see that for $\mathrm{M}=0.7$ gives an optimal cooling effectiveness in the vicinity and downstream of injections until a distance of $\mathrm{X} / \mathrm{D}=1.3$. On the other hand, a lower injection rate such as the case of $\mathrm{M}=0.3$ decreases the effectiveness, which is resulted of the low injection rates of cooling fluid that induce mixtures with the boundary layer of main flow, and thereby preventing the good cooling of the blade. The same observation can be noted for the injection rate 
$\mathrm{M}=1.4$; a slight decrease in cooling is observed compared to injection rate $\mathrm{M}=0.7$, and this is due to the high velocities of injection which results in small detachments of jets of the wall, thus promoting the reduction of cooling effectiveness. There is an offset the results for $\mathrm{M}=1.4$ and the other results. This offset is explained by the presence of counter-rotating vortices which decreases the cooling effectiveness. Beyond of the position $\mathrm{X} / \mathrm{D}=1.3$, the film cooling effectiveness become higher with the upper values of $\mathrm{M}$.

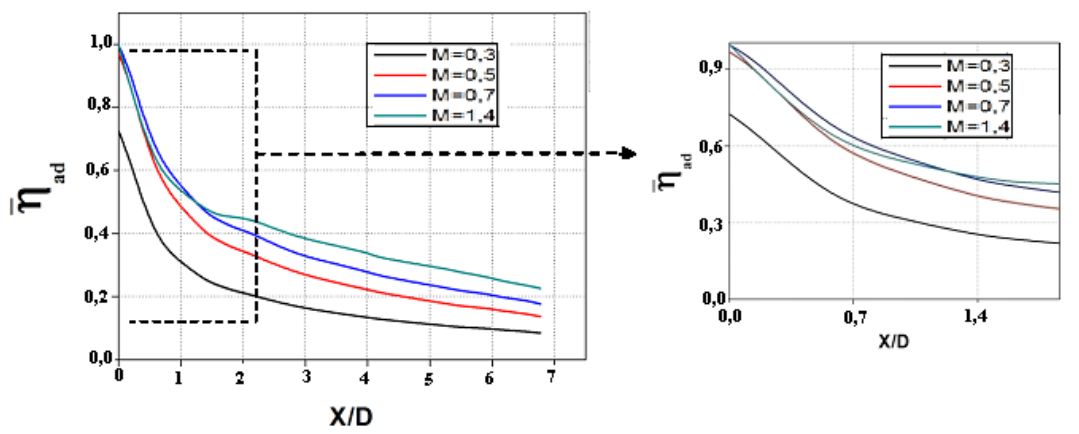

Figure 11. Longitudinal distribution of the average cooling effectiveness according to the injection rate.

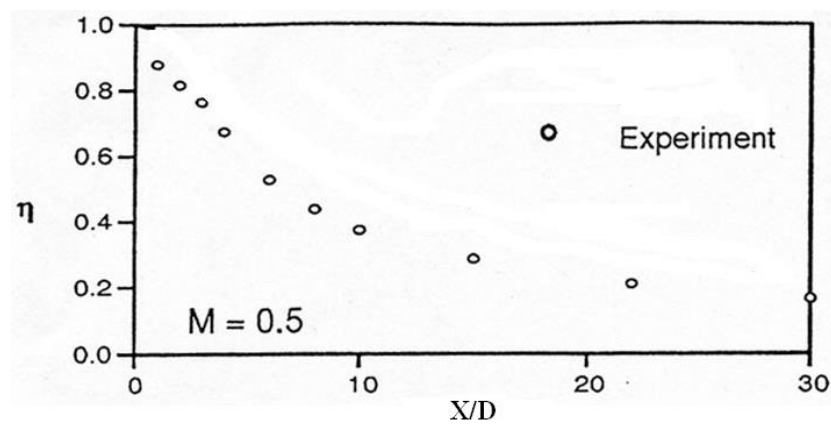

Figure 12. Experimental centerline effectiveness data of Walters and Leylek [16] $(M=0.5)$.

This geometry corresponds to a real blade of a gas turbine. We have no experimental data and it is very difficult to obtain results under real engine condition experiments. To validate our results, we present a global comparison of our results. This comparison relates to the average cooling effectiveness for the injection rate (M $=0.5$ ) with those of Walter and Leylek [16] (figure 12). Walter and Leylek have compared Numerical results to experimental data for the case of a row of three-dimensional, inclined jets with length-to-diameter ratios similar to a realistic film-cooling application. The three-dimensional test case was examined for three different blowing ratios, from 0.5 to 2.0. All of the simulations had a density ratio of 2.0, and an injection angle of $35^{\circ}$. We present a quantitative comparison of Figure $11(\mathrm{M}=0.5)$ with Figure 12. Although the SST turbulence model is relatively better in precision, they show a great disagreement with the experimental results. The reason for the apparent difference between the CFD simulation and experimental work may be due to the anisotropy of the cooling flow field of the film. Furthermore, this is not the same working conditions and this is not the same study domain.

For different blowing ratios, optimum cooling efficiency depends on the position, shape and angle of injection of holes.

Abu Talib et al. [17] show that blowing ratio of 0.64 provides a better cooling protection compared to the other blowing ratios tested (0.5-0.94) for an angle of inclination of $45^{\circ}$. Zhu Huiren et al. [18] show that the critical blowing ratio is 1.3 for the dustpan- and fan-shaped holes, 0.7 for the round holes. The blowing ratio ranges from 0.3 to 2.0 .

We also note that the increase in injection rate causes the elongation of the film cooling as seen in Figure 13. This can explain a greater blooming of film cooling of blade until the attenuating of the latter.

Figure 14 shows the velocity and temperature fields on a plane passing through the injection hole for the four injection rate $(\mathrm{M}=0.3,0.5,0.7$ and 1.4). These figures show the interaction between the jet and the main flow. For the lower injection rate $\mathrm{M}=0.3$, we see that the main flow penetrates in the hole forming inside a 
mixture which is engendered subsequently to bad cooling. For cases $M=0.5$ and $M=0.7$, the jets are deflected energetically in the sense of the main flow with no formation of a recirculation zone. However, for the case $\mathrm{M}=1.4$, the jet managed to get into the main flow and contributed to the formation of a small recirculation zone, thus reducing the protective qualities just downstream of the injection hole. The examination of isotherms confirms previous results, namely that the higher the injection rate, the greater the blooming of the jet into the main flow is more important.

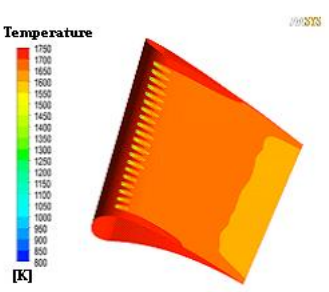

$M=0.3$

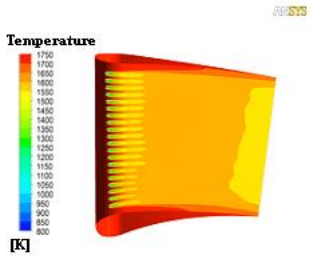

$M=0.7$

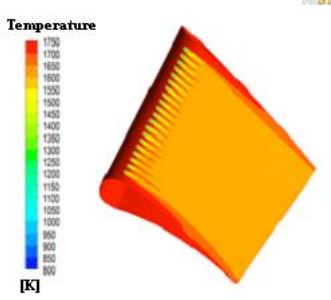

$M=0.5$

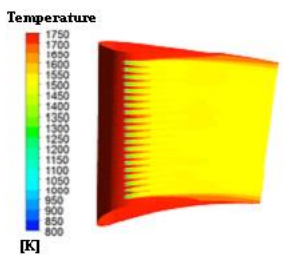

$M=14$

Figure 13. Temperature distribution on the blade for different injection rate $\mathrm{M}(0.3,0.5,0.7$ and 1.4)

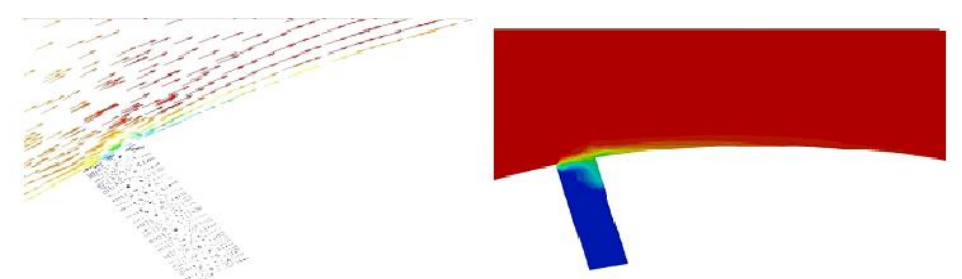

$\mathbf{M}=\mathbf{0 . 3}$

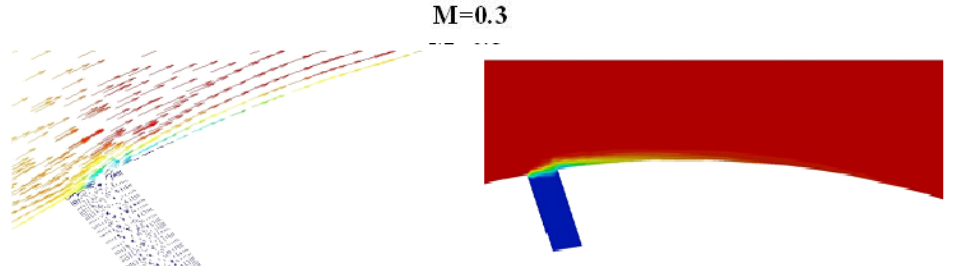

$\mathrm{M}=\mathbf{0 . 5}$

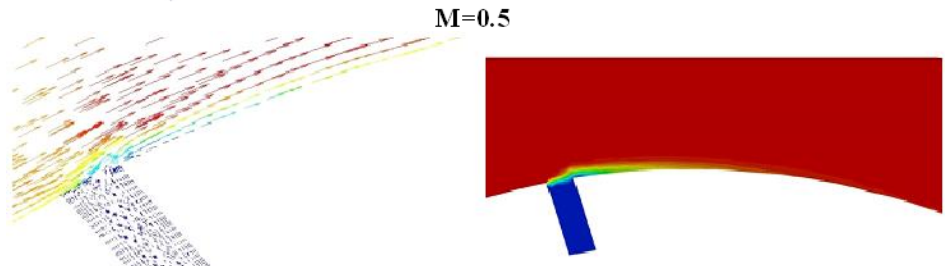

$\mathbf{M}=\mathbf{0 . 7}$

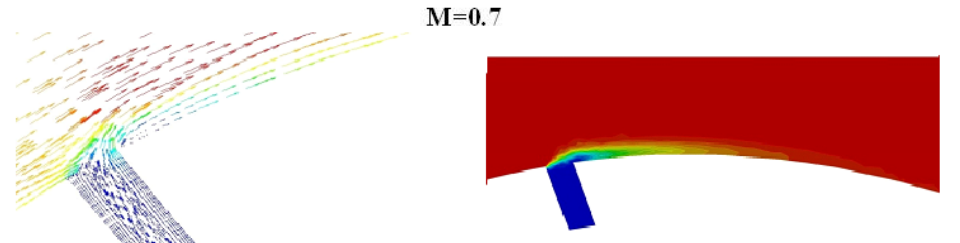

$\mathbf{M}=\mathbf{1 . 4}$

Figure 14. Vectors velocities and distribution of the temperature on a longitudinal plane passing in the middle of the injection hole $\mathrm{M}=0.3,0.5,0.7$ and 1.4. 
Figures 15 represent the velocity field for the two injection rate and for the positions $(\mathrm{X} / \mathrm{D}=1$ and $\mathrm{X} / \mathrm{D}=1.5)$. It is noted that the effect of increasing the injection rate is characterized by the elevation of the height of the boundary layer, which decreases gradually by going away from the injection hole. The visualization of vectors velocities shows the absence of the vortex structures for $\mathrm{X} / \mathrm{D}=1$ for $\mathrm{M}=0.7$. However, for $\mathrm{M}=1.4$, we observe the appearance of a vortex structure to $\mathrm{X} / \mathrm{D}=1.5$.

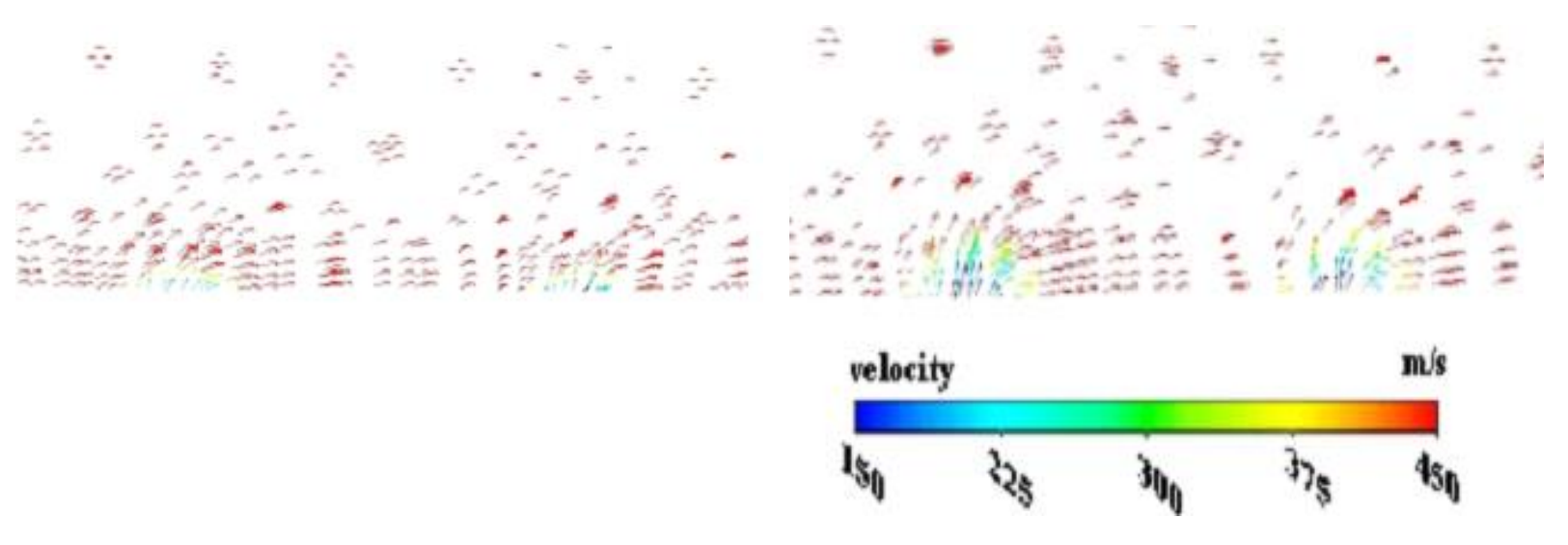

$\mathbf{M}=\mathbf{0 . 7}$

$M=1.4$

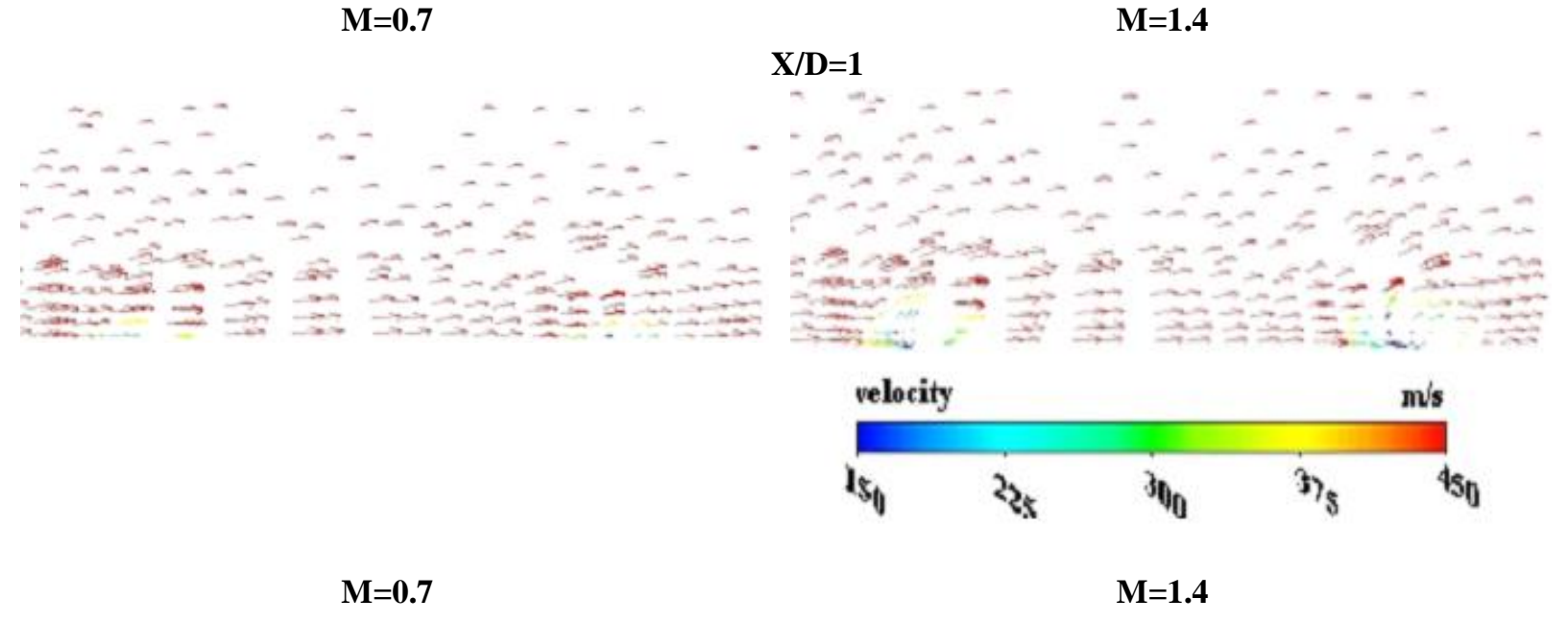

$\mathrm{X} / \mathrm{D}=\mathbf{1 . 5}$

Figure 15. Contours of velocity fields for two injection rate $M(0.7 ; 1.4)$ for $X / D=1$ and 1.5

\section{CONCLUSION}

The results obtained in this study are more or less consistent compared to previous studies presented by various researchers and have shown that the structure of the jet is fairly complex, of three-dimensional nature and is strongly influenced by several parameters such as injection rate.

The numerical simulations presented in this work have shown that the injections of higher rate contributes to a better cooling of the wall (if the inclination angle is small) to a certain value from which the vortices appear. For higher injection rates $(\mathrm{M}=1.4)$, we observed the appearance of a vortex structure which are unfavorable in the cooling process but only downstream of the injection hole. In our work we found that $\mathrm{M}=0.7$ given an optimal cooling efficiency in the vicinity and downstream of injections. The injection rate $\mathrm{M}=0.7$ offers better thermal protection in the zone of the injection holes location.

\section{REFERENCES}

[1] A. Chernobrovkin and B. Lakshminarayana: Numerical simulation and aerothermal physics of leading edge film cooling. J. Power. Energy. Vol. 213, No. 2, (1999), p. 103-118.

[2] A. Hoda and S. Acharya: Predictions of a film coolant jet in cross-flow with different turbulence models. ASME J. Turbomach. Vol. 122, No. 3, (2000), p. 558-569.

[3] I. Gartshore, M. Salcudean and I. Hassan: Film cooling injection hole geometry: hole shape comparison for compound cooling orientation. AIAA J. Vol. 39, No. 8, (2001), p. 1493-1499. 
[4] R.J. Goldstein: Advances in Heat Transfer, Academic Press, New York. pp. 321-379, (1971)

[5] R.J. Margason: Fifty Years of Jet in Cross-Flow Research, AGARDCP- 534, (1993)

[6] N.W.Foster NW and D. Lampard: The flow and film cooling effectiveness following injection through a row of holes. Journal of Engineering for Power, vol. 107, (1985), p. 105-110.

[7] H. Nasir, S.V. Ekkad and S. Acharya: Effect of compound angle injection on flat surface film cooling with large streamwise injection angle. Experimental Thermal and Fluid Science, vol. 25, (2001), p. 23-29.

[8] Xiao Yang and Tang Hong-wu: Numerical simulation of a horizontal momentum jet in cross-flow. Journal of Hydrodynamics, Ser. B, Vol. 18, (2006), p. 118-125.

[9] Fan Jing-yu, Wang Dao-zeng and Zhang Yan: Three-dimensional mean and turbulence characteristics of an impinging density jet in a confined crossflow in near field. Journal of Hydrodynamics, Ser. B, Vol. 16, No. 6, (2004), p. 737-742.

[10] N. Fadéla, A. Azzi, G. Theodoridis and A. Jubran: Reynolds stress transport modelling of film cooling at the leading edge of a symmetrical turbine blade model. Heat Transfer Engineering. Vol. 29, No. 11, (2008), p. 950960.

[11] J.P. Bons, C.D. MacArthur and R.B. Rivir: The Effect of High Free-StreamTurbulence on Film Cooling Effectiveness. ASME Journal of Turbomachinery, Vol. 118, (1996), p. 814-825.

[12] E. E. Halila, D. T. Lenahan and T. T. Thomas: Energy efficient engine high pressure turbine test hardware detailed design report. NASA-CR-167955 (1982).

[13] F. R. Menter: Two-Equation Eddy-Viscosity Turbulence Models for Engineering Applications. AIAA Journal, Vol. 32, No. 8, (1994), p. 1598-1605.

[14] R. F. Warming and Richard M. Beam: Upwind Second-Order Difference Schemes and Applications in Aerodynamic Flows. AIAA Journal, Vol. 14, No. 9, (1976), p. 1241-1249.J.-

[15] M. Miao and H. K. Ching: Numerical simulation of film-cooling concave plate as coolant jet passes through two rows of holes with various orientations of coolant flow. International Journal of Heat and Mass Transfer 49 (2006), p. 557-574

[16] D. K. Walters and J. H. Leylek: A Detailed Analysis of Film-Cooling Physics: Part I-Streamwise Injection with Cylindrical Holes," ASME Journal of Turbomachinery, Vol. 122, (2000), p. 102-112.

[17] A. R. Abu Talib, A. A. Jaafar, A. S. Mokhtar, Mohd Saiah H. R., I. Abd. Rahim and M.S. Abdul Karim : Effects of blowing ratio on the heat transfer coefficient distribution downstream of a single film cooling hole. International Journal of Engineering and Technology, Vol. 3, No.1, (2006), p. 37-46

[18] Zhu Huiren, Xu Duchun, Guo Tao and Liu Songling: Effects of film cooling hole shape on heat transfer. Heat Transfer, Asian Research, Vol.33, Issue 2, (2004), p. 73-80. 